\title{
Surface Micropatterning of Uniaxially Oriented Polyethylene Films Using Interference Holography for Strain Sensors
}

\author{
Lihua Shen, ${ }^{\dagger}$ Sarah S. D. Lafleur, ${ }^{\dagger}$ Simon J. A. Houben, ${ }^{\dagger}$ Jeffrey N. Murphy, ${ }^{\dagger}$ John R. Severn, ${ }^{\dagger,}$ \\ and Cees W. M. Bastiaansen ${ }^{*},, \S(0)$
}

\author{
${ }^{\dagger}$ Laboratory of Functional Organic Materials and Devices, Eindhoven University of Technology, P.O. Box 513, 5600 MB Eindhoven, \\ The Netherlands \\ ${ }^{\ddagger}$ DSM Materials Science Center, NL-6160 MD Geleen, The Netherlands \\ ${ }^{\S}$ School of Engineering and Materials Science, Queen Mary University of London, London E1 4NS, U.K.
}

\section{Supporting Information}

ABSTRACT: A new procedure is presented for direct generation of surface micropatterns on uniaxially oriented polyethylene (PE) films using interference holography with a nanosecond pulsed laser. An ultraviolet absorber, 2- $(2 \mathrm{H}-$ benzotriazol-2-yl)-4,6-di-tert-pentylphenol (BZT) is incorporated into PE prior to stretching to generate absorption at the wavelength of the laser. Illumination with an interference pattern in the absorption band of BZT leads to an obvious height variation in the exposed regions and consequently relief gratings are generated. The height in the exposed regions is strongly dependent on the angle between the grating direction and the film orientation direction. This phenomenon is attributed to a combination of events such as melting, entropic contraction, recrystallization, thermal evaporation of $\mathrm{BZT}$, and anisotropic thermal conductivity. It is shown that the relief height increases with increasing BZT concentration and exhibits a linear dependence on the energy dose above a certain threshold. Additionally, the oriented PE films with the surface

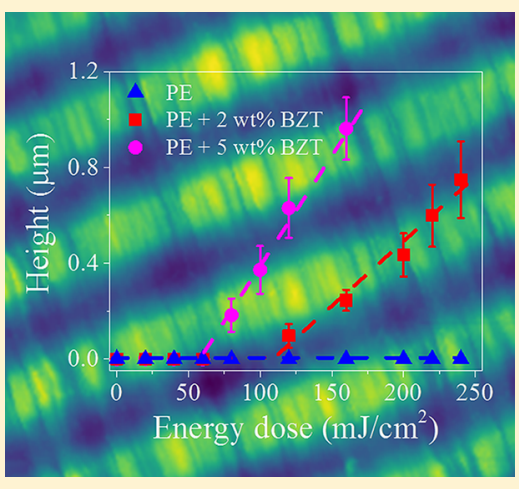
micropatterns are explored for strain sensors. The results demonstrate that small strains below $10 \%$ are monitored accurately in tensile deformation of the micropatterned, oriented PE films which makes these films potentially useful as strain sensors.

\section{INTRODUCTION}

The ability to micropattern polymeric materials with predesigned surface structures is of great significance in a wide range of applications including sensors, ${ }^{1,2}$ tissue engineering, ${ }^{3-6}$ lab-on-a-chip (LoC) devices, ${ }^{7}$ and biofouling., ${ }^{8,9}$ A large variety of micropatterning strategies have been developed based on soft lithography, ${ }^{10-12}$ holography, ${ }^{13,14}$ spatial light modulator (SLM), ${ }^{15}$ inkjet printing, ${ }^{16-19}$ direct laser interference patterning (DLIP), ${ }^{20-25}$ and micro- and nanoprinting techniques. $^{26-28}$ Among these technologies, interference holography is a facile, inexpensive, and maskless technology to generate periodic structures in a photoresist layer. ${ }^{29,30}$ Typically, a laser beam is split into two beams with identical linear polarizations from which an interference pattern is created with a pitch determined by the angle between two interfering laser beams. ${ }^{31}$ In the past, both negative and positive photoresist materials were employed extensively in interference holography to generate the microstructures in isotropic monomers and polymers and etching procedures are commonly employed to develop the surface relief structures. ${ }^{30,32-34}$ For instance, Netti et al. ${ }^{35}$ reported an effective and inexpensive technique to fabricate switchable holographic patterns in a light-sensitive azobenzene-based polymer which is used to guide cell adhesion and orientation.
Normally, highly oriented polyethylene is produced by uniaxial stretching in the solid state, i.e., at temperatures close to but below the melting temperature. ${ }^{36,37}$ The solid state stretching procedure leads to a high degree of molecular orientation and chain extension in polyethylene in the deformation direction, and hence enhances its mechanical properties including Young's modulus and tensile strength. ${ }^{38,39}$ Uniaxially oriented polyethylenes are now used in load-bearing applications such as cranes and elevator ropes, ${ }^{40}$ and detecting strain in these ropes upon tensile deformation is important with respect to predicting their mechanical failure. In the present study, a facile and straightforward method is developed to monitor strain in uniaxially oriented polyethylene films upon tensile deformation based on surface relief patterns. The approach to developing surface micropatterns consists of exposing the film to an interference pattern using a pulsed laser with a wavelength of $355 \mathrm{~nm}$. To form the micropatterns, an appropriate amount of 2-(2H-benzotriazol-2-yl)-4,6-di-tertpentylphenol (BZT) is incorporated into polyethylene as a photoabsorber prior to solid state stretching. Surface micropatterns with well-controlled relief heights and grating pitches

Received: October 16, 2017

Revised: December 5, 2017

Published: December 5, 2017 
are successfully obtained on the surface of the oriented polyethylene film without employing etching procedures. The underlying mechanism for the generation of the micropatterns is further investigated, and the feasibility of monitoring small strains in the patterned, oriented polyethylene film during tensile deformation is discussed.

\section{EXPERIMENTAL SECTION}

Materials. High density polyethylene was obtained from Borealis (Burghausen, Germany) with number- and weight-average molecular weights of 37 and $134 \mathrm{~kg} / \mathrm{mol}$, respectively. 2-(2H-Benzotriazol-2-yl)4,6-di-tert-pentylphenol (BZT) was purchased from BASF (Germany). Silicon oil was purchased from Thermo Fisher Scientific Inc. (The Netherlands). All reagents were used directly as received without further purification. ZAP-IT photosensitive paper was obtained from Edmund Optics Inc. (USA).

Preparation of Specimens. Uniaxially oriented polyethylene films containing 2 and $5 \mathrm{wt} \%$ BZT were produced according to a procedure described previously. ${ }^{41}$ The 2 wt $\%$ BZT and 5 wt \% BZT were mixed with polyethylene in a corotating twin screw extruder at $160{ }^{\circ} \mathrm{C}$ for $10 \mathrm{~min}$. Subsequently, polyethylene sheets containing BZT with a thickness of $0.3 \mathrm{~mm}$ were produced by compression molding at $160{ }^{\circ} \mathrm{C}$ for $5 \mathrm{~min}$, followed by cooling to room temperature in a watercooled press. The compression-molded sheets were subsequently solid state stretched 10 times at $80{ }^{\circ} \mathrm{C}$ in air using a Zwick Z100 tensile tester at a crosshead speed of $100 \mathrm{~mm} / \mathrm{min}$. Uniaxially oriented polyethylene films with a thickness of approximately $100 \mu \mathrm{m}$ were obtained. The draw ratio $(\lambda)$ was determined from the displacement of ink marks inscribed on the sheets prior to stretching at an interval of 2 $\mathrm{mm}$.

Micropatterning of uniaxially oriented polyethylene (PE) films was performed using a pulsed Nd:YAG laser coupled to second and third harmonic modules emitting 4 ns pulses at a repetition rate of $10 \mathrm{~Hz}$ and a wavelength of $355 \mathrm{~nm}$. Linear polarized light with a vertical polarization direction was used, and a schematic diagram of the experimental setup is shown in Figure S1. In most cases, the film orientation direction is horizontal, which is perpendicular to the grating direction. The energy dose is tuned by rotating a half-wave plate in combination with a beam splitter which determines the intensity of the laser.

The recording angles $(2 \theta)$ between two interference beams were 5.2, 2.0, 1.2, and $0.8^{\circ}$, which correspond to grating pitches of approximately $4,10,16$, and $26 \mu \mathrm{m}$, respectively. The interference patterns have a diameter of $8 \mathrm{~mm}$ and the intensity is rather nonuniform, as shown using an exposed photosensitive paper in Figure S2. In this particular case, the photosensitive paper was exposed to an interference pattern with an energy dose of $120 \mathrm{~mJ} / \mathrm{cm}^{2}$. The energy dose was measured using a Thorlabs PM100D power meter (Germany) at the film position in Figure S1.

The influence of the angle $(\alpha)$ between the grating direction and the film orientation direction on the height of the relief gratings was investigated by rotating the film with respect to the grating direction in a range between 0 and $90^{\circ}$. In this case, the oriented PE film with 5 wt $\%$ BZT was exposed to an interference pattern with a period of approximately $10 \mu \mathrm{m}$ at a constant energy dose of $120 \mathrm{~mJ} / \mathrm{cm}^{2}$.

For reference, a direct laser writing procedure was also performed using the same pulsed Nd:YAG laser, as shown in Figure S3. For direct laser writing, the oriented film was mounted on an $x-y$ translation stage and the film orientation direction was always horizontal, i.e., parallel to the $x$-axis of the translation stage. The film was exposed to a single and focused beam with an energy dose of $120 \mathrm{~mJ} / \mathrm{cm}^{2}$ per pulse, and the film moved along the $x$-axis at a constant speed of $1 \mathrm{~mm} / \mathrm{s}$. A straight line with a width of $0.6 \mathrm{~mm}$ was generated along the film orientation direction. Prior to exposure, whether to an interference pattern or a direct laser writing procedure, the oriented PE films were always placed between glass slides and silicon oil was used to reduce the surface light scattering which results from the fibrillar structure of the solid state drawn films. ${ }^{41}$ The reflections between the glass, the silicon oil, and oriented polyethylene are weak below $1 \%$ according to the Fresnel equations and Snell's law, and they are therefore ignored in this study. After recording of the patterns, the oil on the film surface was removed by rinsing with isopropyl alcohol.

Strain Sensors. An oriented polyethylene film with relief gratings with a pitch of $8.5 \mu \mathrm{m}$ was mounted between clamps in a Zwick Z100 tensile tester, as shown in Figure S4. A $10 \mathrm{~mW} \mathrm{He-Ne} \mathrm{laser} \mathrm{with} \mathrm{a}$ wavelength of $632.8 \mathrm{~nm}$ and a beam diameter of $0.65 \mathrm{~mm}$ was used for generating a diffraction pattern. The laser was aligned with the propagation direction of light perpendicular to the film surface, and the light polarization direction was parallel to the film orientation direction. The diffraction pattern was projected on a screen with a millimeter grid, and a film-to-screen distance $(d)$ of $22 \mathrm{~cm}$ was used. Subsequently, strain was imposed on the films at $1 \%$ intervals, between 0 and $10 \%$ along the film orientation direction. The change in the position of the first diffraction order was measured using the grid paper. In all cases, at least three specimens were investigated and the average displacement of the first diffraction order and the standard deviation were calculated. Repeated testing of the strain sensors was carried out at room temperature. The patterned oriented film with a grating pitch of $8.5 \mu \mathrm{m}$ was repeatedly loaded and unloaded to a strain of $6 \%$, and the distance between two first diffraction orders was measured. In this case, 10 cycles were used. To investigate the temperature influence of the strain sensors, similar testing procedures were used, except that the measurements were performed in a Zwick Z100 tensile tester equipped with a temperature-controllable oven. The oriented polyethylene films were deformed at strains of 6 and $8 \%$ and at a temperature between 20 and $60{ }^{\circ} \mathrm{C}$.

Characterization. The surface morphology of the oriented polyethylene films with a thin gold layer was analyzed using a JSMIT100 scanning electron microscope (SEM) (Japan) operating in a high vacuum mode and at an accelerating voltage of $10 \mathrm{kV}$. Optical microscopy images were obtained using a Leica CTR 6000 microscope. Differential scanning calorimetry (DSC) was performed at a constant heating and cooling rate of $10{ }^{\circ} \mathrm{C} / \mathrm{min}$ under nitrogen flow using a DSC Q1000 instrument. Thermogravimetric analyses (TGA) were performed in a TA Q500 instrument at a constant heating rate of $10{ }^{\circ} \mathrm{C} / \mathrm{min}$ and at a constant air flow rate of $50 \mathrm{~mL} /$ min. Small-angle light scattering (SALS) was carried out according to a procedure reported previously. ${ }^{42}$ The film was located between two crossed polarizers with the film orientation direction parallel to the analyzer (Hv). The height of the relief gratings was measured using a DektakXT surface profiler with a tip diameter of $2 \mu \mathrm{m}$ and a force of 3 mg. At least five different positions were measured to estimate the average relief height on the film surface. Atomic force microscopy (AFM) measurements were performed using a Solver P47H scanning probe microscope equipped with a SMENA-B detecting head (NTMDT Ltd., Moscow, Russia) in a tapping mode and at a frequency of $320 \mathrm{kHz}$. Aluminum coated NCHV-A tips (Bruker) with a tip length of $4 \mu \mathrm{m}$ were used. Postprocessing of the raw AFM data was performed using Gwyddion ${ }^{43}$ and ImageJ, and the average and median height profiles of relief gratings on the film surface were obtained perpendicular to the grating direction.

\section{RESULTS}

Previously, it was found that the BZT molecules in the uniaxially oriented polyethylene (PE) film are nondichroic and they are not incorporated in the orthorhombic unit cell of polyethylene. $^{41}$ In other words, the BZT molecules are mainly concentrated in the noncrystalline regions between the microfibrils of the oriented PE. ${ }^{41,44}$ More importantly, it was found that transparent, oriented PE films are produced by introducing the BZT molecules into PE prior to solid state stretching. The transparent appearance makes it possible to monitor strains of these oriented PE films using a laser beam in the transmission mode, which will be discussed later.

The oriented PE films without and with 5 wt \% BZT were exposed to an interference pattern at an identical energy dose of $120 \mathrm{~mJ} / \mathrm{cm}^{2}$. In the oriented PE film without BZT, only 
fibrillar structures are observed (Figure 1A), which form during solid state drawing of the melt-crystallized PE and there is no
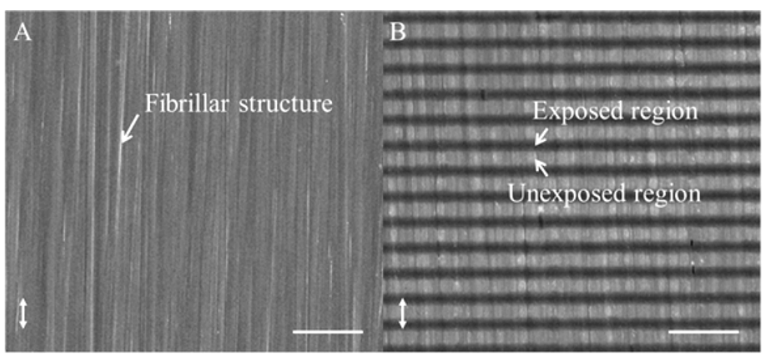

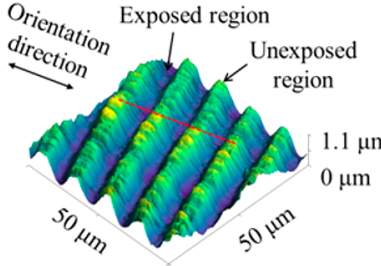

$\mathrm{C}$

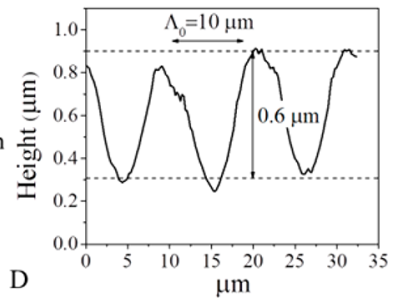

Figure 1. SEM images of oriented PE films (A) without and (B) with 5 wt $\%$ BZT after illumination with an interference pattern at an energy dose of $120 \mathrm{~mJ} / \mathrm{cm}^{2}$. The arrows indicate the film orientation direction, and the scale bar is $25 \mu \mathrm{m}$. (C) AFM 3-D image of the oriented PE film containing 5 wt \% BZT after illumination with an interference pattern $\left(\Lambda_{0}=9.5 \mu \mathrm{m}\right)$ at an energy dose of $120 \mathrm{~mJ} / \mathrm{cm}^{2}$ and (D) the relief height profile along the red dashed line in image $C$.

grating on the film surface. In sharp contrast, in the oriented PE film containing $5 \mathrm{wt} \% \mathrm{BZT}$, periodic gratings with a pitch of $\Lambda_{0}=9.5 \pm 0.3 \mu \mathrm{m}$ are successfully generated with the grating direction perpendicular to the film orientation direction (Figure 1B). The fibrillar structures in the unexposed regions are again observed, and this is in contrast to the exposed regions which exhibit nonfibrillar structures.

The three-dimensional (3-D) AFM image in Figure 1C also indicates that the microfibrils completely disappear in the exposed regions. The unexposed regions are higher than the exposed regions, which indicates that a height reduction has taken place in the exposed regions. The relief grating possesses a height of $0.6 \mu \mathrm{m}$, as shown in Figure 1D.

The influence of energy dose on the height of the relief gratings on the surface of oriented PE film was also explored at an identical grating pitch $\left(\Lambda_{0}=9.5 \mu \mathrm{m}\right)$ with the grating direction perpendicular to the film orientation direction. The results in Figure 2 illustrate that a linear relationship exists between the relief height and the energy dose above a certain threshold. The relief height linearly increases to approximately $1 \mu \mathrm{m}$ as the energy dose increases to $160 \mathrm{~mJ} / \mathrm{cm}^{2}$ for the oriented PE film with 5 wt \% BZT. At energy doses higher than $160 \mathrm{~mJ} / \mathrm{cm}^{2}$, it was observed that the exposed region becomes slightly brown, which indicates the onset of degradation of the oriented polyethylene. A similar linear relationship between the relief height and the energy dose above a threshold is also observed for the oriented PE film with 2 wt \% BZT (Figure 2). The slope of the dose-response curve is, however, reduced quite significantly and the threshold below which relief gratings are not observed is higher.

The pitch of relief gratings was varied by changing the angle between the interfering laser beams (see the Experimental Section), and pitches of $4.5 \pm 0.2,9.5 \pm 0.3,16.3 \pm 0.8$, and $26.4 \pm 0.5 \mu \mathrm{m}$ are successfully obtained (Figure S5).

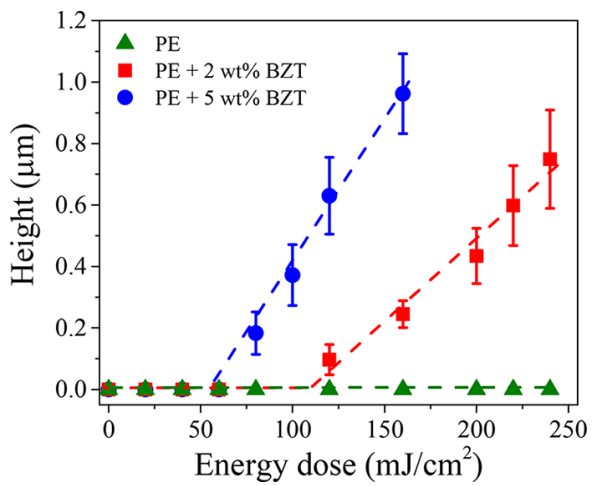

Figure 2. Height of relief gratings $\left(\Lambda_{0}=9.5 \mu \mathrm{m}\right)$ as a function of energy dose for oriented PE containing various BZT contents (measured by Dektak). The lines are used as a visual guide.

Additionally, the angle $(\alpha)$ between the film orientation direction and the grating direction was adjusted by rotating the film between 0 and $90^{\circ}$ (see the Experimental Section). As shown in Figure 3, relief gratings are successfully generated on the film surface at all angles (see also Figure S6 for AFM images).

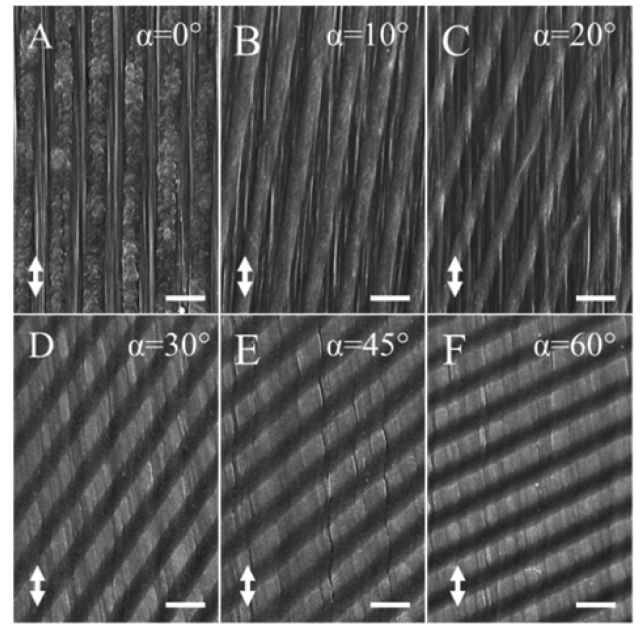

Figure 3. (A-F) SEM images of oriented PE films containing 5 wt \% BZT after illumination with an interference pattern $\left(\Lambda_{0}=9.5 \mu \mathrm{m}\right)$ at an energy dose of $120 \mathrm{~mJ} / \mathrm{cm}^{2}$ and at various angles $(\alpha)$ between the grating direction and the film orientation direction. Arrows indicate the film orientation direction. Scale bar: $10 \mu \mathrm{m}$.

AFM images in Figure 4 indicate that the average relief height is highly dependent on the angle $(\alpha)$ between the grating and the orientation direction. The height of the relief structures is averaged because microfibrils in the unexposed regions create variations in relief height perpendicular to the grating direction (Figure S7). Moreover, the profiles in Figure $\mathrm{S} 7$ also indicate that there is no obvious variation in the grating pitch. The exposed regions are higher than the unexposed regions at $\alpha=0^{\circ}$ (Figure 4A,C), and this is in sharp contrast to the relief gratings at $\alpha=90^{\circ}$ (Figure 4B,D).

In Figure 5, the average height of the relief gratings using both AFM and a Dektak surface profiler (see the Experimental Section) is plotted as a function of the angle $(\alpha)$ between the grating direction and the film orientation direction. The results show an excellent agreement in the measurements of the average relief height between AFM and the Dektak surface 

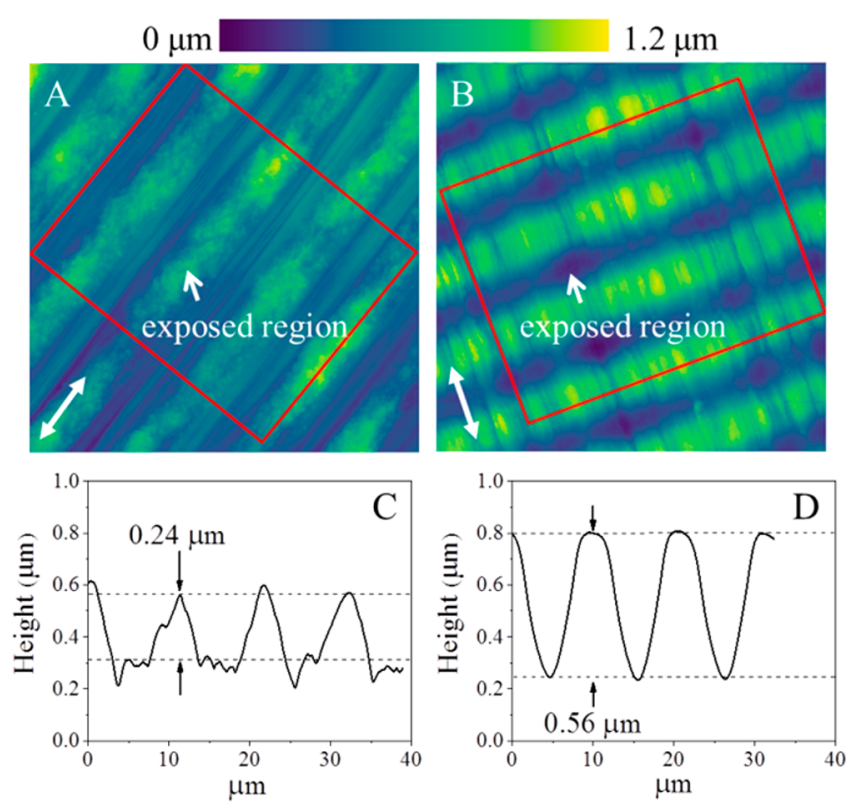

Figure 4. AFM images $(50 \mu \mathrm{m} \times 50 \mu \mathrm{m})$ of oriented PE films containing 5 wt $\%$ BZT after illumination with an interference pattern $\left(\Lambda_{0}=9.5 \mu \mathrm{m}\right)$ at an energy dose of $120 \mathrm{~mJ} / \mathrm{cm}^{2}$ and at various angles $(\alpha)$ between the grating direction and the film orientation direction: (A) $\alpha=0^{\circ}$ and (B) $\alpha=90^{\circ}$. (C) and (D) show the corresponding average height profiles in the marked regions of $(A)$ and $(B)$ (see the Experimental Section). Arrows indicate the film orientation direction.

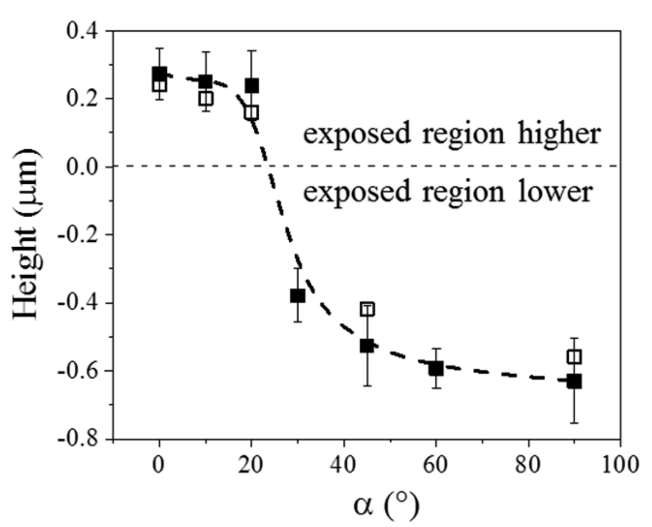

Figure 5. Height of relief gratings as a function of the angle $(\alpha)$ between the grating direction and the film orientation direction of oriented PE films containing 5 wt \% BZT after illumination with an interference pattern $\left(\Lambda_{0}=9.5 \mu \mathrm{m}\right)$ at an energy dose of $120 \mathrm{~mJ} / \mathrm{cm}^{2}$. Solid symbols, data from Dektak surface profiler; empty symbols, data from AFM.

profiler. It is found that at an angle $(\alpha)$ between 30 and $90^{\circ}$, the exposed regions are lower than the unexposed regions. The average relief height by the Dektak surface profiler decreases from $0.63 \pm 0.12$ to $0.38 \pm 0.08 \mu \mathrm{m}$ as the angle $(\alpha)$ changes from 90 to $30^{\circ}$. The exposed regions become higher than the unexposed regions at $\alpha$ equal to or below $20^{\circ}$. The benzotriazole (BZT)-based UV absorber is well-known for its photochemical properties. ${ }^{45-48}$ By illumination with UV light in the absorption band at $355 \mathrm{~nm}$, the non-hydrogen-bonding [N] state of BZT absorbs photo energy. Subsequently, the transoid $[\mathrm{N}] *$ state is converted into the cisoid, hydrogenbonding $[\mathrm{H}]$ state via isomerization. Alternatively, the $[\mathrm{N}] *$ state may proceed to the $[\mathrm{N}]$ ground state by transferring energy to the $[\mathrm{H}]$ ground state, which then dissipates the photoexcitation energy as heat by a proton transfer mechanism. The thermal energy results in a temperature increase in the exposed region. To demonstrate this hypothesis, the oriented PE film containing 5 wt \% BZT was directly exposed to a single and focused laser beam at an energy dose of $120 \mathrm{~mJ} / \mathrm{cm}^{2}$ per pulse. The film was mounted on an $x-y$ stage (see the Experimental Section). A line with a width of $0.6 \mathrm{~mm}$ along the film orientation direction was generated using a direct laser writing procedure, as shown in Figure S8A,B.

The microscopy image under crossed polarizers in Figure $\mathrm{S} 8 \mathrm{~B}$ indicates that isotropic crystalline structures form in the written region. The result is verified using small-angle light scattering (SALS) in the so-called $\mathrm{Hv}$ mode where a four-lobe cloverleaf pattern indicates the formation of spherulitic structures in the written region (Figure S8C). It is therefore concluded that the absorption of UV light results in a temperature increase in the written region which leads to local melting of the oriented PE and subsequently recrystallization upon cooling at room temperature. In other words, the temperature in the written region at least rises higher than the melting point of the oriented $\mathrm{PE}\left(T_{\mathrm{m}}=140{ }^{\circ} \mathrm{C}\right)$ (Figure S9). It seems reasonable to assume that local melting also occurs during interference holography at an identical energy dose. For the patterned PE film at $\alpha=90^{\circ}$, AFM images and the corresponding height profiles in Figure S10 indicate that the height variation in the exposed regions along the grating direction from the microfibrils becomes less apparent, which is attributed to the melting and subsequently recrystallization upon illumination.

Generally, upon exposure to an interference pattern, the BZT induces a UV light intensity gradient in the cross section of the oriented PE films. The relative light intensity of the UV light at the position $z(\mu \mathrm{m})$ in the thickness direction is expressed by the equation based on Lambert-Beer's law:

$$
\frac{I_{(z)}}{I_{\mathrm{o}}}=10^{-\mu z c}
$$

where $\mu$ is the absorption coefficient (for BZT, $\mu \approx 0.01$ (wt $\%)^{-1} \mu \mathrm{m}^{-1}$ ), $c$ is the BZT concentration (wt \%), $I_{\mathrm{o}}$ is the initial intensity of the incident laser, and $I_{(z)}$ is the intensity at the position $(z)$ in the film thickness direction. In Figure S11, it is shown that the UV light is almost completely absorbed in the top layer at the positions of $z=30 \mu \mathrm{m}$ and $z=70 \mu \mathrm{m}$ in the thickness direction of the oriented PE film containing 5 and 2 wt \% BZT, respectively. For the oriented PE film containing 5 wt \% BZT upon exposure to an interference pattern with a high energy dose of $160 \mathrm{~mJ} / \mathrm{cm}^{2}$, an average height of approximately $1 \mu \mathrm{m}$ of the relief gratings was observed (Figure 2) which corresponds to $\sim 3 \%$ of height decrease in the exposed region based on the depth of the UV light in the film $(z=30 \mu \mathrm{m})$ (Figure S11). Therefore, a volume decrease of less than $3 \%$ is estimated in the exposed region. This volume decrease might be partially attributed to the thermal evaporation of BZT at a high temperature. In Figure S12, the onset of weight loss of BZT is observed at a temperature of $140{ }^{\circ} \mathrm{C}$ and 95 wt \% of BZT evaporates at a temperature of $247^{\circ} \mathrm{C}$.

In the present study, an oriented polyethylene film with a relief grating $\left(\Lambda_{1}=8.5 \mu \mathrm{m}\right)$ was used to measure strains in the film upon tensile deformation. In Figure S13, a laser beam $(\lambda=$ $632.8 \mathrm{~nm}$ ) with its propagation direction of light perpendicular to the film surface is used. In this case, multiple diffraction 

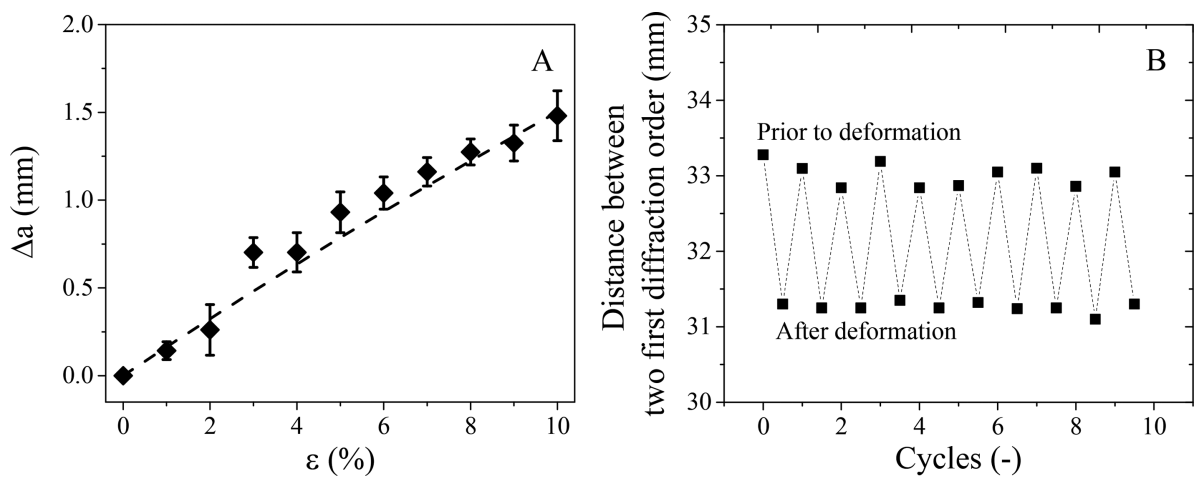

Figure 6. (A) Displacement $\left(\Delta a=a_{1}-a_{11}\right)$ of the first diffraction order as a function of strain $(\varepsilon)$ and (B) multiple-cycle tests with repeated loading-unloading at $6 \%$ strain. The patterned oriented film possesses a grating pitch of $\Lambda_{1}=8.5 \mu \mathrm{m}$. The dashed line in (A) represents the theoretical calculation based on eqs $1-3$, and the dashed line in (B) is used as a visual guide.

orders are generated (Figure S14), which indicates that thin gratings are obtained in the so-called Raman-Nath regime.

For simplification, only the zero $\left(m_{0}\right)$ and the first diffraction orders $\left( \pm m_{1}\right.$ or $\left.\pm m_{11}\right)$ are shown in Figure S13. When a strain $(\varepsilon)$ in the film orientation direction is imposed (Figure S13), the initial grating period $\Lambda_{1}$ increases to $\Lambda_{11}$, where $\Lambda_{11}$ is equal to $(1+\varepsilon) \Lambda_{1}$. The diffraction angle $\theta_{m_{11}}$ of the first diffraction order $\left(m_{11}\right)$ after deformation is expressed by

$$
\Lambda_{11} \sin \theta_{m_{11}}=\Lambda_{1}(1+\varepsilon) \sin \theta_{m_{11}}=\lambda
$$

Similarly, the distance $\left(a_{11}\right)$ between the $m_{0}$ and the $m_{11}$ diffraction orders after deformation in Figure S13 is obtained by

$$
a_{11}=d \tan \theta_{m_{11}}
$$

Based on eqs 1 and 2, the displacement $\left(\Delta a=a_{1}-a_{11}\right)$ of the first diffraction order prior to and after deformation is predicted at a given strain $(\varepsilon)$

$$
\Delta a=a_{1}-a_{11}=d\left(\tan \theta_{\mathrm{m}_{1}}-\tan \theta_{\mathrm{m}_{11}}\right)
$$

where $a_{1}$ is the distance between the $m_{0}$ and the $m_{1}$ diffraction orders prior to deformation.

An excellent agreement is observed between the experimental data and the theoretical predictions based on eqs $1-3$ (Figure 6). A displacement of $1.48 \pm 0.14 \mathrm{~mm}$ of the first diffraction order corresponds to a strain of $10 \%$ of the patterned oriented PE film. The results in Figure 6B demonstrate an excellent reversibility of the strain sensors at a strain of $6 \%$. The temperature dependence of the relief structures with a grating pitch of $\Lambda_{1}=8.5 \mu \mathrm{m}$ was also investigated at strains of 6 and $8 \%$ (Figure S15). The results indicate that the temperature hardly influences the accuracy of the sensors.

\section{DISCUSSION}

A new procedure is reported for generation of surface micropatterns on uniaxially oriented polyethylene films using interference holography. These micropatterns are rather inhomogeneously distributed across the film surface. This inhomogeneity is attributed to an inhomogeneous intensity distribution of the interference pattern which was generated with a pulsed laser (Figure S2). More importantly, it is demonstrated that the BZT used in this study functions like a linear photoresist; i.e., the height of the relief gratings increases linearly with increasing energy dose (Figure 2) above a certain threshold, which consequently results in an inhomogeneity in the height of the relief gratings on the film surface. The inhomogeneity issue was circumvented by measuring the most intense regions in Figure S2, and at least five regions were measured using the Dektak surface profiler to reflect the actual height of relief gratings. The micropatterns are probably generated based on a combination of local melting/ recrystallization of the uniaxially oriented polyethylene as well as thermal evaporation of BZT in the exposed regions. Normally, melting the oriented polyethylene leads to entropy-driven contraction which is expected to result in a height increase while thermal evaporation of BZT is expected to decrease the height of the relief structures in the exposed regions. It is also well-established that uniaxially oriented polyethylenes have a highly anisotropic thermal conductivity. ${ }^{49,50}$ The anisotropy in thermal conductivity of the oriented high density polyethylene at a $\lambda=10$ is around 30 at room temperature. $^{37}$ It is anticipated that this anisotropic thermal conductivity leads to a variation in the entropy-driven contraction of the oriented polyethylene and thereby contributes to the angle-dependent height of the relief structure.

\section{CONCLUSIONS}

In this study, we report the generation of micropatterns on the surface of uniaxially oriented PE film using interference holography. The method is based on a BZT-induced transition from photo energy to heat by illumination with an interference pattern. This process leads to local melting and entropy-driven contraction of the oriented PE, and thermal evaporation of the $\mathrm{BZT}$ in the exposed regions, which is accompanied by a height change and formation of the micropatterns and this without using etching procedures. In the case of the energy dose above the threshold, the actual height of the relief gratings on the film surface is dependent on not only the initial BZT concentration in the film and the energy dose used, but also the angle between the film orientation direction and the grating direction. It is also shown that the micropatterned films can be used as strain sensors with a comparatively high accuracy. The strain sensors are reversible and rather insensitive to temperature.

\section{ASSOCIATED CONTENT}

\section{S Supporting Information}

The Supporting Information is available free of charge on the ACS Publications website at DOI: 10.1021/acs.langmuir.7b03599. 
Schematic diagram of the experimental setup used for interference holography and direct laser writing, schematic illustration of the experimental setup for strain sensors, photograph of photosensitive paper after illumination, SEM images, AFM images, microscopy images, SALS pattern, DSC curves, relative light intensity, TGA curves, schematic illustration of strain sensors, multiple diffraction patterns, and temperature influence on strain sensing (PDF)

\section{AUTHOR INFORMATION}

\section{Corresponding Author}

*E-mail: C.W.M.Bastiaansen@tue.nl.

ORCID $\odot$

Cees W. M. Bastiaansen: 0000-0003-1198-7528

Author Contributions

L.S. and S.S.D.L. contributed equally to this work.

Notes

The authors declare no competing financial interest.

\section{ACKNOWLEDGMENTS}

L.S. acknowledges financial support by the China Scholarship Council (CSC), and S.S.D.L. thanks DSM Dyneema BV, The Netherlands, for support.

\section{REFERENCES}

(1) Tan, J. L.; Tien, J.; Pirone, D. M.; Gray, D. S.; Bhadriraju, K.; Chen, C. S. Cells Lying on a Bed of Microneedles: An Approach to Isolate Mechanical Force. Proc. Natl. Acad. Sci. U. S. A. 2003, 100, 1484-1489.

(2) Kuwabara, K.; Ogino, M.; Motowaki, S.; Miyauchi, A. Fluorescence Measurements of Nanopillars Fabricated by HighAspect-Ratio Nanoprint Technology. Microelectron. Eng. 2004, 73$74,752-756$.

(3) Chen, C. S.; Mrksich, M.; Huang, S.; Whitesides, G. M.; Ingber, D. E. Geometric Control of Cell Life and Death. Science 1997, 276, $1425-1428$.

(4) Brown, R. A.; Wiseman, M.; Chuo, C. B.; Cheema, U.; Nazhat, S. N. Ultrarapid Engineering of Biomimetic Materials and Tissues: Fabrication of Nano- and Microstructures by Plastic Compression. Adv. Funct. Mater. 2005, 15, 1762-1770.

(5) Hoque, M. E.; Hutmacher, D. W.; Feng, W.; Li, S.; Huang, M.H.; Vert, M.; Wong, Y. S. Fabrication Using a Rapid Prototyping System and in Vitro Characterization of PEG-PCL-PLA Scaffolds for Tissue Engineering. J. Biomater. Sci., Polym. Ed. 2005, 16, 1595-1610.

(6) Martina, M.; Subramanyam, G.; Weaver, J. C.; Hutmacher, D. W.; Morse, D. E.; Valiyaveettil, S. Developing Macroporous Bicontinuous Materials as Scaffolds for Tissue Engineering. Biomaterials 2005, 26, 5609-5616.

(7) Bianco, V.; Mandracchia, B.; Marchesano, V.; Pagliarulo, V.; Olivieri, F.; Coppola, S.; Paturzo, M.; Ferraro, P. Endowing a Plain Fluidic Chip with Micro-Optics: A Holographic Microscope Slide. Light: Sci. Appl. 2017, 6, e17055.

(8) Granhag, L. M.; Finlay, J. A.; Jonsson, P. R.; Callow, J. A.; Callow, M. E. Roughness-Dependent Removal of Settled Spores of the Green Alga Ulva (Syn. Enteromorpha) Exposed to Hydrodynamic Forces from a Water Jet. Biofouling 2004, 20, 117-122.

(9) Hoipkemeier-Wilson, L.; Schumacher, J. F.; Carman, M. L.; Gibson, A. L.; Feinberg, A. W.; Callow, M. E.; Finlay, J. A.; Callow, J. A.; Brennan, A. B. Antifouling Potential of Lubricious, MicroEngineered, PDMS Elastomers Against Zoospores of the Green Fouling Alga Ulva (Enteromorpha). Biofouling 2004, 20, 53-63.

(10) Xia, Y.; Whitesides, G. M. Soft Lithography. Angew. Chem., Int. Ed. 1998, 37, 550-575.
(11) Grilli, S.; Vespini, V.; Ferraro, P. Surface-Charge Lithography for Direct PDMS Micro-Patterning. Langmuir 2008, 24, 13262-13265.

(12) Qin, D.; Xia, Y.; Whitesides, G. M. Soft Lithography for Microand Nanoscale Patterning. Nat. Protoc. 2010, 5, 491-502.

(13) Wathuthanthri, I.; Liu, Y.; Du, K.; Xu, W.; Choi, C. H. Simple Holographic Patterning for High-Aspect-Ratio Three-Dimensional Nanostructures with Large Coverage Area. Adv. Funct. Mater. 2013, 23, 608-618.

(14) Ning, H.; Pikul, J. H.; Zhang, R.; Li, X.; Xu, S.; Wang, J.; Rogers, J. A.; King, W. P.; Braun, P. V. Holographic Patterning of HighPerformance On-Chip 3D Lithium-Ion Microbatteries. Proc. Natl. Acad. Sci. U. S. A. 2015, 112, 6573-6578.

(15) Miccio, L.; Memmolo, P.; Grilli, S.; Ferraro, P. All-Optical Microfuidic Chips for Reconfgurable Dielectrophoretic Trapping through SLM Light Induced Patterning. Lab Chip 2012, 12, 44494454.

(16) Yakovlev, A. V.; Milichko, V. A.; Vinogradov, V. V.; Vinogradov, A. V. Sol-Gel Assisted Inkjet Hologram Patterning. Adv. Funct. Mater. 2015, 25, 7375-7380.

(17) Yakovlev, A. V.; Pidko, E. A.; Vinogradov, A. V. Inkjet Printing of Transparent Sol-Gel Computer Generated Holograms. Opt. Mater. Express 2016, 6 (12), 3794-3803.

(18) Furasova, A. D.; Ivanovski, V.; Yakovlev, A. V.; Milichko, V. A.; Vinogradov, V. V.; Vinogradov, A. V. Inkjet Fabrication of Highly Efficient Luminescent Eu-Doped $\mathrm{ZrO}_{2}$ Nanostructures. Nanoscale 2017, 9, 13069-13078.

(19) Eremeeva, E. A.; Yakovlev, A. V.; Pidko, E. A.; Vinogradov, A. V. UV-Curable Hybrid Organic-Inorganic Composite Inks with a High Refractive Index for Printing Interference Images and Holograms. J. Mater. Chem. C 2017, 5, 5487-5493.

(20) Montelongo, Y.; Yetisen, A. K.; Butt, H.; Yun, S.-H. Reconfigurable Optical Assembly of Nanostructures. Nat. Commun. 2016, 7, 12002.

(21) AlQattan, B.; Butt, H.; Sabouri, A.; Yetisen, A. K.; Ahmed, R.; Mahmoodi, N. Holographic Direct Pulsed Laser Writing of TwoDimensional Nanostructures. RSC Adv. 2016, 6, 111269-111275.

(22) Guo, J. J.; Liu, X. Y.; Jiang, N.; Yetisen, A. K.; Yuk, H.; Yang, C. X.; Khademhosseini, A.; Zhao, X. H.; Yun, S. H. Highly Stretchable, Strain Sensing Hydrogel Optical Fibers. Adv. Mater. 2016, 28, 1024410249.

(23) Ahmed, R.; Yetisen, A. K.; Yun, S. H.; Butt, H. Color-Selective Holographic Retroreflector Array for Sensing Applications. Light: Sci. Appl. 2017, 6, e16214.

(24) Ahmed, R.; Yetisen, A. K.; El Khoury, A.; Butt, H. Printable Ink Lenses, Diffusers, and 2D Gratings. Nanoscale 2017, 9, 266-276.

(25) Jiang, N.; Butt, H.; Montelongo, Y.; Liu, F.; Afewerki, S.; Ying, G.-L.; Dai, Q.; Yun, S.-H.; Yetisen, A. K. Laser Interference Lithography for the Nanofabrication of Stimuli-Responsive Bragg Stacks. Adv. Funct. Mater. 2017, 1702715.

(26) Kumar, A.; Whitesides, G. M. Features of Gold Having Micrometer to Centimeter Dimensions Can Be Formed through a Combination of Stamping with an Elastomeric Stamp and an Alkanethiol "ink" followed by Chemical Etching. Appl. Phys. Lett. 1993, 63, 2002-2004.

(27) Lackowski, W. M.; Ghosh, P.; Crooks, R. M. Micron-Scale Patterning of Hyperbranched Polymer Films by Micro-Contact Printing. J. Am. Chem. Soc. 1999, 121, 1419-1420.

(28) Nirmala Grace, A.; Pandian, K. A Polypyrrole/Polymethylene Pattern on Gold Using a Micro-Contact Printing Technique. J. Solid State Electrochem. 2003, 7, 296-300.

(29) Lai, N. D.; Liang, W. P.; Lin, J. H.; Hsu, C. C.; Lin, C. H. Fabrication of Two- and Three-Dimensional Periodic Structures by Multi-Exposure of Two-Beam Interference Technique. Opt. Express 2005, 13, 9605-9611.

(30) Prenen, A. M.; van der Werf, J. C. A.; Bastiaansen, C. W. M.; Broer, D. J. Monodisperse, Polymeric Nano- and Microsieves Produced with Interference Holography. Adv. Mater. 2009, 21, $1751-1755$. 
(31) Brueck, S. R. J. Optical and Interferometric LithographyNanotechnology Enablers. Proc. IEEE 2005, 93, 1704-1721.

(32) Xia, D.; Ku, Z.; Lee, S. C.; Brueck, S. R. J. Nanostructures and Functional Materials Fabricated by Interferometric Lithography. Adv. Mater. 2011, 23, 147-179.

(33) Anderson, E. H.; Horwitz, C. M.; Smith, H. I. Holographic Lithography with Thick Photoresist. Appl. Phys. Lett. 1983, 43, 874875.

(34) Niggemann, M.; Blaesi, B.; Boerner, V.; Gombert, A.; Klicker, M.; Kuebler, V.; Lalanne, P.; Wittwer, V. Periodic Microstructures for Large Area Applications Generated by Holography. Proc. SPIE 2001, 4438, 108-115.

(35) Rianna, C.; Calabuig, A.; Ventre, M.; Cavalli, S.; Pagliarulo, V.; Grilli, S.; Ferraro, P.; Netti, P. A. Reversible Holographic Patterns on Azopolymers for Guiding Cell Adhesion and Orientation. ACS Appl. Mater. Interfaces 2015, 7, 16984-16991.

(36) Capaccio, G.; Ward, I. M. Preparation of Ultra-High Modulus Linear Polyethylenes; Effect of Molecular Weight and Molecular Weight Distribution on Drawing Behaviour and Mechanical Properties. Polymer 1974, 15, 233-238.

(37) Capaccio, G.; Crompton, T. A.; Ward, I. M. Drawing Behavior of Linear Polyethylene. II. Effect of Draw Temperature and Molecular Weight on Draw Ratio and Modulus. J. Polym. Sci., Polym. Phys. Ed. 1980, 18, 301-309.

(38) Hallam, M. A.; Cansfield, D. L. M.; Ward, I. M.; Pollard, G. A Study of The Effect of Molecular Weight on The Tensile Strength of Ultra-High Modulus Polyethylenes. J. Mater. Sci. 1986, 21, 41994205.

(39) Smith, P.; Lemstra, P. J.; Pijpers, J. P. L. Tensile Strength of Highly Oriented Polyethylene. II. Effect of Molecular Weight Distribution. J. Polym. Sci., Polym. Phys. Ed. 1982, 20, 2229-2241.

(40) Homepage Dyneema. https://www.dsm.com/products/ dyneema/en_AU/applications/ropes-lines-and-slings/crane-rope.html (accessed Oct 3, 2017).

(41) Shen, L.; Nickmans, K.; Severn, J.; Bastiaansen, C. W. M. Improving the Transparency of Ultra-Drawn Melt-Crystallized Polyethylenes: Toward High-Modulus/High-Strength Window Application. ACS Appl. Mater. Interfaces 2016, 8, 17549-17554.

(42) Stein, R. S.; Hotta, T. Light Scattering from Oriented Polymer Films. J. Appl. Phys. 1964, 35, 2237-2242.

(43) Necas, D.; Klapetek, P. Gwyddion: an Open-Source Software for SPM Data Analysis. Cent. Eur. J. Phys. 2012, 10, 181-188.

(44) Shen, L.; Severn, J.; Bastiaansen, C. W. M. Improving VisibleLight Transparency of Drawn Melt-Crystallized Linear Polyethylenes: Influence of Molecular Weight Distribution. Macromol. Mater. Eng. 2017, 302, 1700003.

(45) Cortolano, F. P. Antioxidants and UV Stabilizers. A Summary of Their Utilization in PVC. J. Vinyl Technol. 1993, 15, 69-75.

(46) Crawford, J. C. 2-(2-Hydroxyphenyl)-2H-Benzotriazole Ultraviolet Stabilizers. Prog. Polym. Sci. 1999, 24, 7-43.

(47) Allan, D. S.; Maecker, N. L.; Priddy, D. B.; Schrock, N. J. Modeling Photodegradation in Transparent Polymers. Macromolecules 1994, 27, 7621-7629.

(48) Jiang, Y.; Wu, S.; Sustic, A.; Xi, F.; Vogl, O. Functional Polymers. LVI. Photochemical Behavior of 2(2-Hydroxyphenyl)2HBenzotriazole Derivatives 4. Spectroscopic Study of Ultraviolet Absorbers with More than One 2(2-Hydroxyphenyl)2H-Benzotrlazole Group and More than One Ortho-Hydroxy Group in the Mol. Polym. Bull. 1988, 20, 169-176.

(49) Choy, C. L.; Luk, W. H.; Chen, F. C. Thermal Conductivity of Highly Oriented Polyethylene. Polymer 1978, 19, 155-162.

(50) Wang, X.; Ho, V.; Segalman, R. A.; Cahill, D. G. Thermal Conductivity of High Modulus Polymer Fibers. Macromolecules 2013, 46, 4937-4943. 